\title{
Reactivity of 2-substituted hydrazinecarbothioamides towards tetracyanoethylene and convenient synthesis of (5-amino-2-diazenylthiazolylmethylene)malononitrile derivatives
}

\author{
Alaa A. Hassan, ${ }^{\text {a, } *}$ Nasr K. Mohamed, ${ }^{\text {a }}$ Kamal M. A. El-Shaieb, ${ }^{\text {a }}$ Hendawy N. Tawfeek, ${ }^{a}$ \\ Stefan Bräse, ${ }^{b}$ and Martin Nieger ${ }^{c}$ \\ ${ }^{a}$ Chemistry Department, Faculty of Science, Minia University, 61519 El-Minia, Egypt \\ ${ }^{b}$ Institute of Organic Chemistry, Karlsruhe Institute of Technology, Fritz-Haber-Weg 6, \\ 76131 Karlsruhe, Germany \\ ${ }^{c}$ Laboratory of Inorganic Chemistry, Department of Chemistry, University of Helsinki \\ P.O. Box 55 (A. I. Virtasen aukio 1), 00014 Helsinki, Finland \\ E-mail: alaahassan2001@mu.edu.eg
}

\section{DOI: https://doi.org/10.24820/ark.5550190.p009.872}

\begin{abstract}
2-\{Amino-[5-amino-2-(substituted diazenyl)thiazol-4-yl]methylene $\}$ malononitriles were synthesized from the reaction of 2-substituted hydrazinecarbothioamides with tetracyanoethylene (TCNE) to give tetracyanoethane adduct, followed by heterocyclization afforded the target compounds. The structure of (E)-2-\{amino-[5-amino-2-(phenyldiazenyl)thiazol-4-yl]methylene $\}$ malononitrile was supported by single crystal X-ray crystallography.
\end{abstract}

Keywords: Malononitrile, thiazoles, thiosemicarbazides, tetracyanoethylene, X-ray crystallography

\section{Introduction}

Recently, thiazole derivatives have attracted a great deal of interest due to their low toxicity and broad biological activity. ${ }^{1-5}$ For example, naturally occurring and synthetic thiazoles find applications as antibiotics and anti-inflammatories, ${ }^{4-8}$ while selected aminothiazoles act as inhibitors of human cancer and Alzheimer`s disease. ${ }^{9-11}$

The syntheses of thiazoles and 2-aminothiazoles have been studied extensively, ${ }^{12,13}$ however, the preparation of 5-aminothiazoles has not been so widely reported. Despite this, 5aminothiazoles have received attention in a range of applications from antibiotics ${ }^{14}$ to photosensitizers. $^{15}$ 
A convenient route to 5-amino-4-phenylthiazoles has been developed from $\mathrm{N}$-acylatedglycinamides and Lawesson's reagent via trifluoroacetamides. ${ }^{12}$ A flexible route to 5-aminothiazoles has been developed based on cyclization of diamide adducts, prepared using the Ugi reaction, ${ }^{16-18}$ in presence of Lawesson's reagent. ${ }^{19}$ 5-Amino-3-(substituted benzylidenamino)-2phenylimino-2,3-dihydrothiazole-4-carbonitrile is one of the products which have been isolated from the reaction of aldehyde thiosemicarbazones with tetracyanoethylene (TCNE). ${ }^{20}$

Mesoionic 1,2,4-triazolium-3-thiolate derivatives were synthesized from the reaction of $\mathrm{N}$ substituted 2-phenylhydrazinecarbothioamides with TCNE. ${ }^{21}$

Herein, we report our investigation on the reaction of 2-substituted hydrazinecarbothioamides 1a-e with TCNE 2 (Fig. 1) and compared with the products isolated from the reaction of Nsubstituted 2-phenylhydrazinecarbothioamides with TCNE 2.

\section{Results and Discussion}

Treatment of the hydrazinecarbothioamides 1a-e with TCNE 2 (1.1 equiv) in dry ethyl acetate at room temperature resulted in a pink coloration of the reaction solution which quickly turned reddish orange. Tentatively, the color change observed may be owed to the formation of unstable charge-transfer (CT) complexes. The mixture was stirred and then left to stand for 24 hours at room temperature, resulting in the formation of single products 3a-e in 81-88\% yields (Scheme 1).

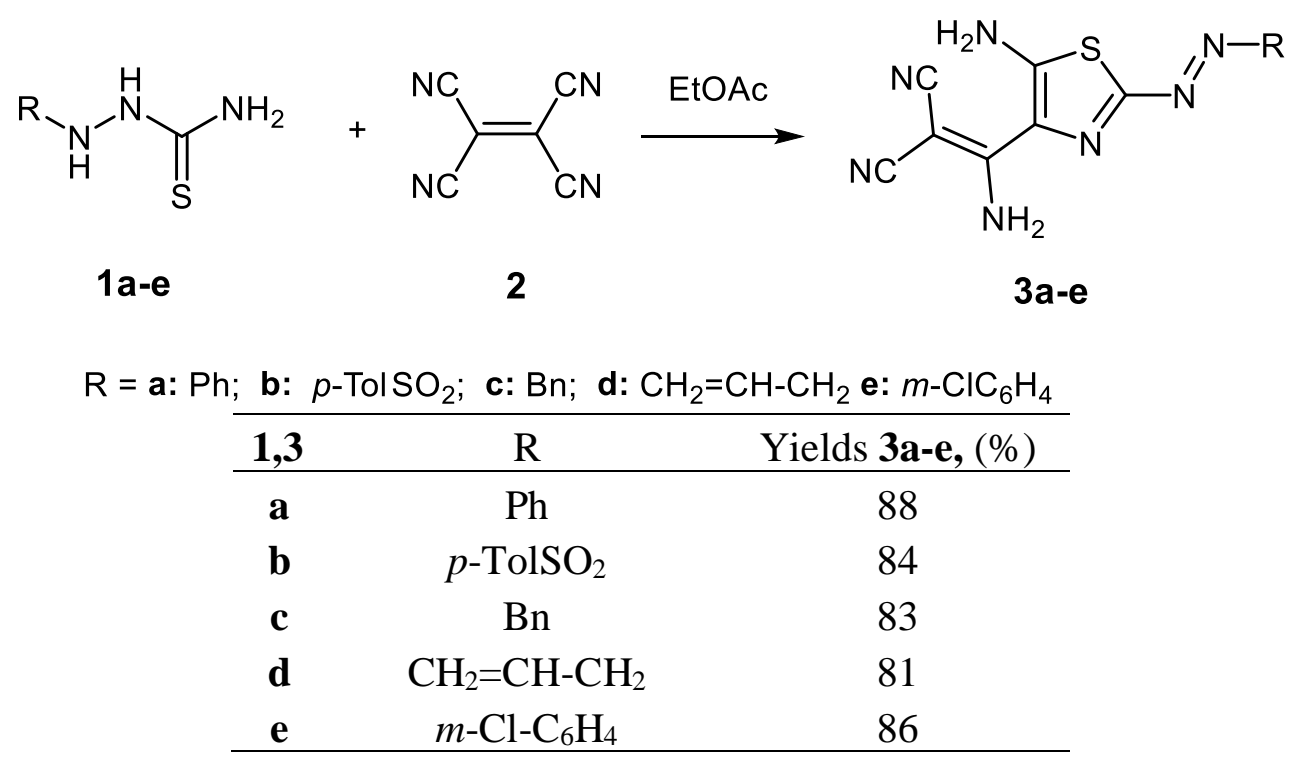

Scheme 1. Reactions of 2-substituted hydrazinecarbothioamides 1a-e with TCNE 2.

The gross formula $\mathrm{C}_{13} \mathrm{H}_{9} \mathrm{~N}_{7} \mathrm{~S}$ represents a product from one molecule of $\mathbf{1 a}$ and one molecule of TCNE 2 without elimination of any atoms. Two $\mathrm{NH}_{2}$ groups are present in ${ }^{1} \mathrm{H} \mathrm{NMR}$ (exchangeable with $\mathrm{D}_{2} \mathrm{O}$ ) as broad signals, the downfield at $10.22 \mathrm{ppm}$ due to $\mathrm{NH}_{2}$ attached to 
vinyl group in 3a, the other $\mathrm{NH}_{2}$ resonate at 7.94 because of $\mathrm{NH}_{2}$ attached to thiazole $\mathrm{C5}$. The aromatic protons observed at 7.83-7.10 ppm. In its ${ }^{13} \mathrm{C}$ NMR spectrum, thiazole $\mathrm{C} 2$, C4 and C5 resonate at 161.2, 150.4 and 152.6, respectively. In the (aminomethylene)malononitrile fragment, of 3a, the dicyanovinyl carbons $\mathrm{C} 2$ and $\mathrm{C} 1$ resonated at 164.4 and $61.6 \mathrm{ppm}$, respectively, and were in accord with the observed trends in the $\delta$ values for $\mathrm{C}$-atoms in push-pull alkenes. ${ }^{22,23}$ Further peaks at $115.6(\mathrm{CN})$, besides the aromatic carbons support the assigned structure.

Absorption bands around 3373-3320 $\mathrm{cm}^{-1}$ relating to $\mathrm{NH}_{2}$ groups appeared in IR spectra of 3ad. The IR spectra of 3a-e showed two sharp absorption bands at 2220-2210 $\mathrm{cm}^{-1}(\mathrm{CN})$ and $1623-$ $1612 \mathrm{~cm}^{-1}$ that assigned to $\mathrm{C}=\mathrm{N}$ vibration. The absence of $\mathrm{C}=\mathrm{S}$ signal in IR and ${ }^{13} \mathrm{C} N \mathrm{NMR}$, also support the structures 3a-e. Moreover, the structure of (E)-2-\{amino-[5-amino-2(phenyldiazenyl)thiazol-4-yl]methylene \}malononitrile 3a has been strongly supported by a single crystal X-ray structure analysis (Figure 1 and Tables S1-S7 in the supplementary data). The asymmetric unit of (E)-2-\{amino-[5-amino-2-(phenyldiazenyl)thiazol-4-yl]methylene $\}$ malononitrile 3a $\left(\mathrm{C}_{13} \mathrm{H}_{9} \mathrm{~N}_{7} \mathrm{~S}\right)$, confirms two independent molecules whose conformations differ primarily in the orientations of phenyl and substituted vinyl groups with respect to the thiazole ring. The X-ray structure analysis confirms a transoid geometry of thiazole and substituted groups with respect to the $\mathrm{N}=\mathrm{N}$ double bond.

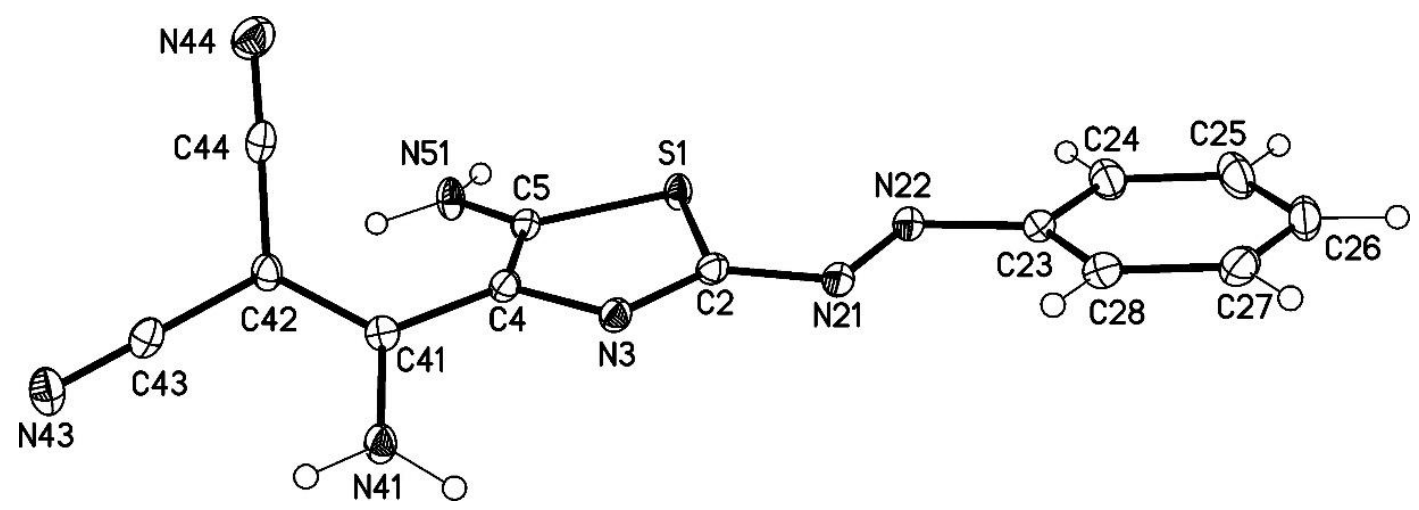

Figure 1. Molecular structure of 3a in the crystal (TIF file). The crystallographic numbering does not reflect the systematic IUPAC numbering.

All bond lengths and angles in compound 3a are normal. The thiazole ring is planar; mean deviation from the $\mathrm{S} 1 / \mathrm{C} 2 / \mathrm{N} 3 / \mathrm{C} 4 / \mathrm{C} 5$ plane $0.019 \AA$. The amino group and the planar phenyldiazenyl substituent are coplanar to the thiazole moiety, while the planar aminomethylenemalononitrile is twisted by $43^{\circ}$ to the thiazole plane.

A rationale for the formation of compounds 3a-d given in Scheme 2. Nucleophilic attack from the terminal $\mathrm{NH}_{2}$ of $\mathbf{1 a - d}$ on the $\mathrm{C}=\mathrm{C}$ double bond of $\mathbf{2}$ to give the tetracyanoethane derivatives $\mathbf{4}$; charge-transfer complexes may, but do not necessarily have to, ${ }^{24}$ play an intermediate role. Intramolecular nucleophilic attack of $\mathrm{SH}$ of $\mathbf{4}$ on the $\mathrm{C} \equiv \mathrm{N}$ triple bond and cyclization to give the intermediate $\mathbf{5}$ followed by the formation of bicyclic $\mathbf{6}$ that can open due to the proton transfer 
from thiazole ring. Compound $\mathbf{6}$ can then rearrange to form $\mathbf{7}$ and $\mathbf{8}$ and finally the highly stable [amino-(5-amino-2-substituted diazenylthiazolyl)methylene]malono-nitriles 3a-e.

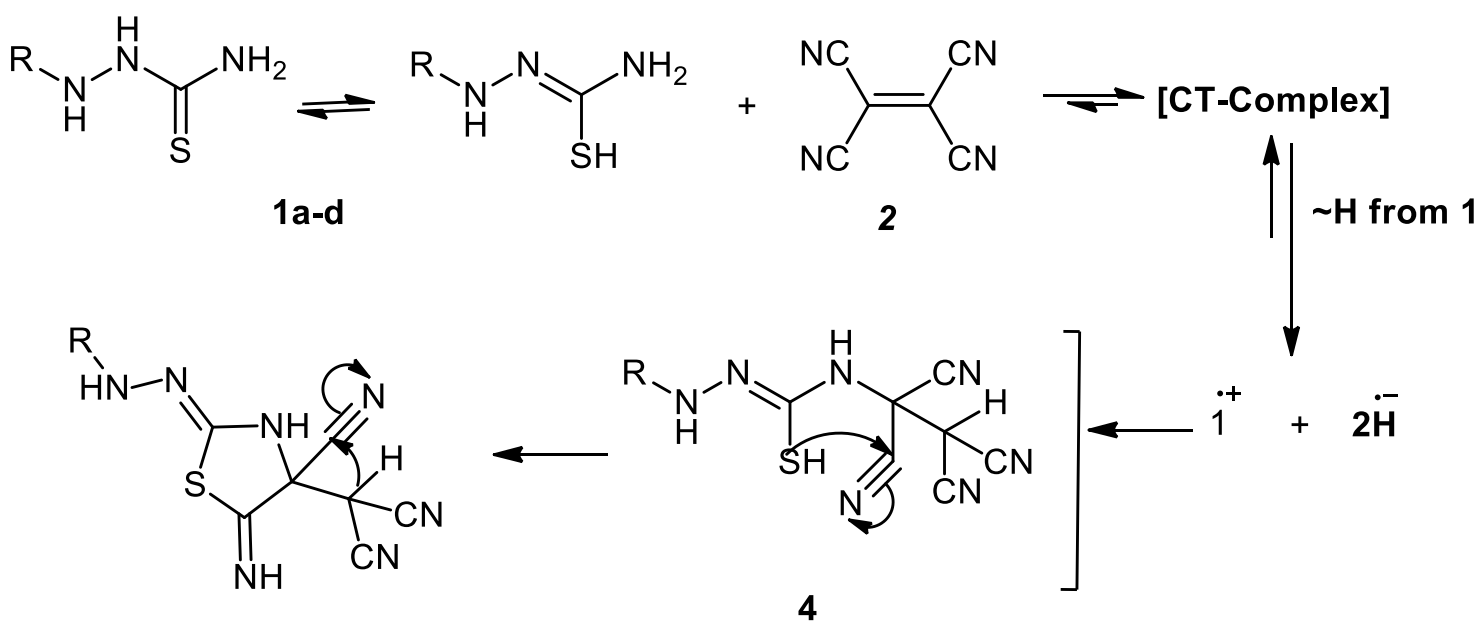

5

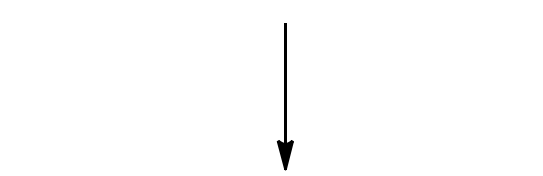

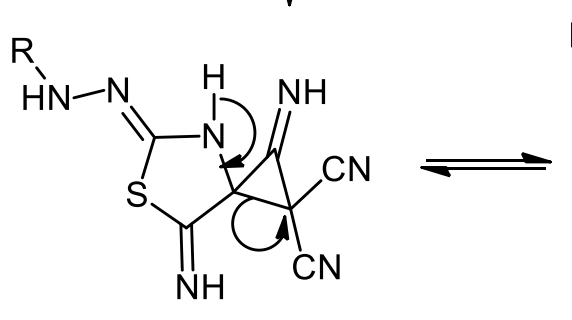

6

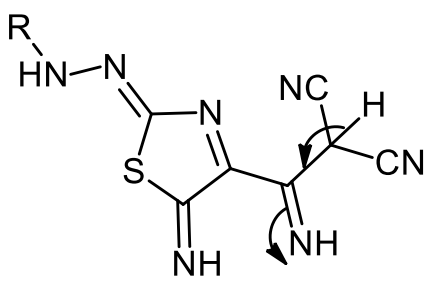

7

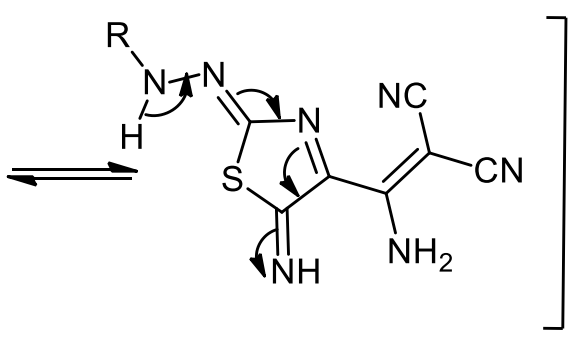

8

廿<smiles>[R]N=Nc1nc(C(N)=C(C#N)C#N)c(N)s1</smiles>

3a-d

Scheme 2: Mechanistic rationale for the formation of compounds 3a-e. 


\section{Conclusions}

In conclusion, novel (diazenylthiazolyl)methylene]malononitriles have been synthesized from the nucleophilic addition reactions of 2-substituted hydrazinecarbothioamides on TCNE. The products were synthesized from readily accessible starting materials using a simple experimental procedure.

\section{Experimental Section}

General. Gallenkamp melting point apparatus was used for determining the melting points; the results are uncorrected. The IR spectra ( $\mathrm{KBr}$ discs technique) were recorded on Alpha, Bruker FTIR and Shimadzu 408 instruments. The ${ }^{1} \mathrm{H}-\mathrm{NMR}(400.13 \mathrm{MHz})$ and ${ }^{13} \mathrm{C}-\mathrm{NMR}(100.6 \mathrm{MHz})$ spectra were determined on a Bruker AM 400 spectrometer; $\mathrm{s}=$ singlet, $\mathrm{m}=$ multiplet, $\mathrm{b}=$ broad. The ${ }^{13} \mathrm{C}$ NMR signals were assigned based on DEPT 135/90 spectra. Chemical shifts are expressed as $\delta$ in parts per million (ppm). The mass spectra $(70 \mathrm{eV}$, electron impact mode) were recorded on a Finnigan MAT 312 instrument. The elemental analyses for C, H, N and S were carried out at the Microanalytical Centre, Cairo University, Egypt using an Elmyer 306. Preparative layer chromatography (PLC) used air-dried $1.0 \mathrm{~mm}$ thick layers of slurry-applied silica gel (Merck $\left.\mathrm{Pf}_{254}\right)$ on glass plates $48 \times 20 \mathrm{~cm}$ using the solvents listed.

\section{Starting materials}

The starting materials 1a-e were prepared following published methods: $\mathbf{1 a},{ }^{25} \mathbf{1 b},{ }^{26} \mathbf{1} \mathbf{c}^{27}, \mathbf{1 d},{ }^{28}$ and $\mathbf{1 e}^{29}$. Tetracyanoethylene (TCNE, 2) was bought from Fluka (USA), recrystallized from chlorobenzene and sublimed before used. Ethyl acetate and toluene were purified according to Vogel $^{30}$ and Organikum, ${ }^{31}$ dried and distilled. Acetonitrile (Merck) was used without further purification.

Reaction of 2-substituted hydrazinecarbothioamides 1a-e with tetracyanoethylene (2). General procedure. A solution of TCNE $2(141 \mathrm{mg}, 1.1 \mathrm{mmol})$ in dry EtOAc $(10 \mathrm{~mL})$, was added dropwise to a solution of 1a-e $(1.0 \mathrm{mmol})$ in dry EtOAc $(15 \mathrm{~mL})$, which causes a spontaneous change of color from yellow to pink and finally to reddish orange. The mixture was stirred for 2 $\mathrm{h}$, then left to stand for $24 \mathrm{~h}$ at room temperature. A red-orange precipitate was formed, filtered and recrystallized from acetonitrile to give pure crystals $3 a-e$. In case of the reaction between $1 \mathrm{~d}$ and TCNE 2, the mixture of the reaction was subjected to PLC and using toluene/ethyl acetate $(5: 1)$ as eluent to give an intense red-orange zone from $3 \mathrm{~d}$. The zone was separated by using acetone and recrystallized from acetonitrile.

(E)-2-\{Amino-5-[amino-2-(phenyldiazenyl)thiazol-4-yl]methylene\}malononitrile (3a). Red crystals $(0.259 \mathrm{~g}, 88 \%), \mathrm{mp} 231-233{ }^{\circ} \mathrm{C}(\mathrm{MeCN}) . \mathrm{IR}: v_{\max }(\mathrm{KBr}) / \mathrm{cm}^{-1} 3373-3342\left(\mathrm{NH}_{2}\right), 2215$ $(\mathrm{CN}), 1620(\mathrm{C}=\mathrm{N}), 1595(\mathrm{Ar}-\mathrm{C}=\mathrm{C}), 1571,1455(\mathrm{~N}=\mathrm{N}) \mathrm{cm}^{-1} . \mathrm{NMR}: \delta_{\mathrm{H}}\left(400 \mathrm{MHz}, \mathrm{DMSO}-d_{6}\right)$ 7.10-7.12 (m, 1H, Ar-H), 7.60-7.62 (m, 2H, Ar-H), 7.80-7-83 (m, 2H, Ar-H), 7.95 (br, s, 2H, NH 2 
attached to thiazole), 10.22 (br, s, $\left.2 \mathrm{H}, \mathrm{NH}_{2}\right) . \delta_{\mathrm{c}}\left(100 \mathrm{MHz}, \mathrm{DMSO}-d_{6}\right) 61.6(\mathrm{C}-\mathrm{C} \equiv \mathrm{N}), 115.6(\mathrm{CN})$, 124.1, 128.6, 129.7 (Ar-CH), 133.0 (Ar-C), 150.5 (thiazole-C4), 152.6 (thiazole-C5), 161.2 (thiazole-C2), $164.4\left(=\mathrm{C}-\mathrm{NH}_{2}\right) . \mathrm{m} / z$ (\%): $295\left(\mathrm{M}^{+}, 76\right), 268$ (14), 190 (16), 105 (37), 77 (100). Anal. Calcd for $\mathrm{C}_{13} \mathrm{H}_{9} \mathrm{~N}_{7} \mathrm{~S}$ (295.32) C, 52.87; H, 3.07; N, 33.20; S, 10.86. Found: C, 53.02; H, $2.94 ; \mathrm{N}, 33.33 ; \mathrm{S}, 10.71 \%$.

(E)-2-\{Amino-5-[amino-2-(4-toluenesulfonyldiazenyl)thiazol-4-yl]methylene\}malononitrile (3b). Red crystals $(0.313 \mathrm{~g}, 84 \%), \mathrm{mp} 255-257^{\circ} \mathrm{C}(\mathrm{MeCN})$. IR: $v_{\max }(\mathrm{KBr}) / \mathrm{cm}^{-1} 3360-3320\left(\mathrm{NH}_{2}\right)$, $2210(\mathrm{CN}), 1617(\mathrm{C}=\mathrm{N}), 1580(\mathrm{Ar}-\mathrm{C}=\mathrm{C}), 1565,1446(\mathrm{~N}=\mathrm{N}) \mathrm{cm}^{-1} . \delta_{\mathrm{H}}\left(400 \mathrm{MHz}, \mathrm{DMSO}-d_{6}\right) 2.42$ (s, 3H, $\left.\mathrm{CH}_{3}\right), 7.52-7.55(\mathrm{~m}, 2 \mathrm{H}, \mathrm{Ar}-\mathrm{H})$ 7.72-7.75 (m, 2H, Ar-H), 7.98 (br, s, 2H, $\mathrm{NH}_{2}$ attached to thiazole), 10.31 (br, s, 2H, NH 2$) . \delta_{\mathrm{C}}\left(100 \mathrm{MHz}, \mathrm{DMSO}-d_{6}\right) 21.2\left(\mathrm{CH}_{3}\right), 61.4(\mathrm{C}-\mathrm{C} \equiv \mathrm{N}), 115.8(\mathrm{CN})$, 125.2, 129.5 (Ar-CH), 135.7, 140.1 (Ar-C), 149.7 (thiazole-C4), 152.6 (thiazole-C5), 161.1 (thiazole-C2), $164.2\left(=\mathrm{C}-\mathrm{NH}_{2}\right) . m / z(\%): 373\left(\mathrm{M}^{+}, 12\right), 346$ (18), 281 (100), 191 (19), 156 (16), 91 (46). Anal. Calcd for $\mathrm{C}_{14} \mathrm{H}_{11} \mathrm{~N}_{7} \mathrm{O}_{2} \mathrm{~S}_{2}$ (373.41) C, 45.03; H, 2.97; N, 26.26; S, 17.17. Found: C, 44.89; H, 3.06; N, 26.12; S, 17.33\%.

(E)-2-\{Amino-[5-amino-2-(benzyldiazenyl)thiazol-4-yl]methylene\}malononitrile (3c). Redorange crystals $(0.256 \mathrm{~g}, 83 \%)$, mp 242-244 ${ }^{\circ} \mathrm{C}(\mathrm{MeCN})$. IR: $v_{\max }(\mathrm{KBr}) / \mathrm{cm}^{-1} 3366-3346\left(\mathrm{NH}_{2}\right)$, $2212(\mathrm{CN}), 1612(\mathrm{C}=\mathrm{N}), 1589(\mathrm{Ar}-\mathrm{C}=\mathrm{C}), 1561,1440(\mathrm{~N}=\mathrm{N}) \mathrm{cm}^{-1} . \delta_{\mathrm{H}}\left(400 \mathrm{MHz}, \mathrm{DMSO}-d_{6}\right) 4.66$ (s, 2H, $\mathrm{CH}_{2} \mathrm{Ph}$ ), 7.04-7.08 (m, 1H, Ar-H), 7.54-7.58 (m, 2H, Ar-H), 7.71-7.76 (m, 2H, Ar-H), 7.98 (br, s, 2H, $\mathrm{NH}_{2}$ attached to thiazole), 10.29 (br, s, 2H, NH 2 ). $\delta_{\mathrm{C}}\left(100 \mathrm{MHz}, \mathrm{DMSO}-d_{6}\right) 52.1$ $\left(\mathrm{CH}_{2} \mathrm{Ph}\right), 61.6(\mathrm{C}-\mathrm{C} \equiv \mathrm{N}), 116.0(\mathrm{CN}), 124.3,128.5,129.1(\mathrm{Ar}-\mathrm{CH}), 133.6$ (Ar-C), 149.7 (thiazoleC4), 152.5 (thiazole-C5), 161.2 (thiazole-C2), 164.4 (=C-NH$) . m / z(\%) 309\left(\mathrm{M}^{+}, 51\right), 282$ (26), 204 (23), 91 (100). Anal. Calcd for $\mathrm{C}_{14} \mathrm{H}_{11} \mathrm{~N}_{7} \mathrm{~S}$ (309.35) C, 54.36; H, 3.58; N, 31.69; S, 10.37. Found: C, 54.22; H, 3.66; N, 31.81; S, 10.23\%.

(E)-2-\{[2-(Allyldiazenyl)-5-aminothiazol-4-yl](amino)methylene\}malononitrile (3d). Redorange crystals $(0.209 \mathrm{~g}, 81 \%), \mathrm{mp} 167-169{ }^{\circ} \mathrm{C}(\mathrm{MeCN}) . v_{\max }(\mathrm{KBr}) / \mathrm{cm}^{-1} 3358-3326\left(\mathrm{NH}_{2}\right), 2210$ $(\mathrm{CN}), 1615(\mathrm{C}=\mathrm{N}), 1558,1438(\mathrm{~N}=\mathrm{N}) \mathrm{cm}^{-1} . \delta_{\mathrm{H}}\left(\mathrm{DMSO}-d_{6}\right) 4.05-4.08\left(\mathrm{~m}, 2 \mathrm{H}\right.$, allyl-CH$\left.{ }_{2} \mathrm{~N}\right), 5.11-$ $5.13\left(\mathrm{~m}, 2 \mathrm{H}\right.$, allyl- $\left.\mathrm{CH}_{2}=\right)$ 5.91-5.94 (m, 1H, allyl-CH=), 7.94 (br, s, 2H, $\mathrm{NH}_{2}$ attached to thiazole), $10.16\left(\mathrm{br}, \mathrm{s}, 2 \mathrm{H}, \mathrm{NH}_{2}\right) . \delta_{\mathrm{c}}\left(\mathrm{DMSO}-d_{6}\right) 49.3\left(\right.$ allyl- $\left.\mathrm{CH}_{2} \mathrm{~N}\right), 60.9(\mathrm{C}-\mathrm{C} \equiv \mathrm{N}), 115.0(\mathrm{CN}), 116.2$ (allyl$\mathrm{CH}_{2}=$ ), 135.4 (allyl-CH=), 149.8 (thiazole-C4), 152.6 (thiazole-C5), 161.9 (thiazole-C2), 164.0 $\left(=\mathrm{C}-\mathrm{NH}_{2}\right) \cdot \mathrm{m} / \mathrm{z}(\%): 259\left(\mathrm{M}^{+}, 21\right), 232(26), 154$ (46), 69 (37), 41 (100). Anal. Calcd for $\mathrm{C}_{10} \mathrm{H}_{9} \mathrm{~N}_{7} \mathrm{~S}$ (259.29) C, 46.32; H, 3.50; N, 37.81; S, 12.37. Found: C, 46.45; H, 3.57; N, 37.93; S, $12.40 \%$.

(E)-2-(Amino\{5-amino-2-[(3-chlorophenyl)diazenyl]thiazol-4-yl\}methylene)malononitrile (3e). Red-orange crystals $(0.282 \mathrm{~g}, 86 \%), \mathrm{mp} 248-250{ }^{\circ} \mathrm{C}(\mathrm{MeCN}) . \mathrm{IR}: v_{\max }(\mathrm{KBr}) / \mathrm{cm}^{-1} 3365-3338$ $\left(\mathrm{NH}_{2}\right), 2220(\mathrm{CN}), 1623(\mathrm{C}=\mathrm{N}), 1583(\mathrm{Ar}-\mathrm{C}=\mathrm{C}), 1569,1450(\mathrm{~N}=\mathrm{N}) \mathrm{cm}^{-1}$. NMR: $\delta_{\mathrm{H}}(400 \mathrm{MHz}$, DMSO-d $d_{6}$ ) 7.55-7.59 (m, 1H, Ar-H), 7.63-7.66 (m, 1H, Ar-H), 7.69-7-80 (m, 2H, Ar-H), 8.01 (br, s, $2 \mathrm{H}, \mathrm{NH}_{2}$ attached to thiazole), $10.28\left(\mathrm{br}, \mathrm{s}, 2 \mathrm{H}, \mathrm{NH}_{2}\right) . \delta_{\mathrm{c}}\left(100 \mathrm{MHz}, \mathrm{DMSO}-d_{6}\right) 61.6(\mathrm{C}-\mathrm{C} \equiv \mathrm{N})$, $115.8(\mathrm{CN}), 125.2,126.3,129.6,130.1$ (Ar-CH), 135.1, 141.2 (Ar-C), 149.7 (thiazole-C4), 153.1 (thiazole-C5), 161.2 (thiazole-C2), $164.5\left(=\mathrm{C}-\mathrm{NH}_{2}\right) . m / z(\%): 329\left(\mathrm{M}^{+}, 36\right), 218$ (28), 190 (61), 139 (43), 111 (100). Anal. Calcd for $\mathrm{C}_{13} \mathrm{H}_{8} \mathrm{ClN}_{7} \mathrm{~S}$ (329.77) C, 47.35; H, 2.45; N, 29.73; S, 9.72. Found: C, 47.42; H, 2.36; N, 29.61; S, 9.83\%. 


\section{Single crystal X-ray structure determination of 3a}

Single crystals were obtained by recrystallization from acetonitrile. The single crystal X-ray diffraction study was carried out on a Bruker D8 Venture diffractometer with Photon100 detector at $123 \mathrm{~K}$ using $\mathrm{CuK} \alpha$ radiation $\left(\lambda=1.54178 \AA\right.$ ) 3a. Direct Methods (SHELXS-97) ${ }^{32}$ were used for structure solution and refinement was carried out using SHELXL-2014 ${ }^{33}$ (full-matrix least-squares on $\mathrm{F}^{2}$ ). Hydrogen atoms were localized by difference electron density determination and refined using a riding model $(\mathrm{H}(\mathrm{N})$ free). A semi-empirical absorption correction was applied.

Compound 3a: $\mathrm{C}_{13} \mathrm{H}_{9} \mathrm{~N}_{7} \mathrm{~S}, \mathrm{M}=295.32 \mathrm{~g} \mathrm{~mol}^{-1}$, red plates, crystal size $0.12 \times 0.08 \times 0.02 \mathrm{~mm}$, triclinic space group, $\mathrm{P}-1$ (no. 2), $\mathrm{a}=7.3780$ (4) $\AA, \mathrm{b}=7.9678$ (5) $\AA, \mathrm{c}=11.9389$ (7) $\AA, \alpha=99.249$ $(2)^{\circ}, \beta=104.589(2)^{\mathrm{o}}, \gamma=100.386(2)^{\mathrm{o}}, \mathrm{V}=652.23(7) \AA^{3}, \mathrm{Z}=2, \mathrm{D}_{\text {calcd }}=1.504 \mathrm{Mg} \mathrm{m}^{3}, \mathrm{~F}(000)=$ $304, \mu=2.256 \mathrm{~mm}^{-1}, \mathrm{~T}=123 \mathrm{~K}, 11384$ measured reflections $\left(2 \theta_{\max }=144^{\circ}\right), 2540$ independent reflections $\left[\mathrm{R}_{\text {int }}=0.030\right.$ ], 202 parameters, 4 restraints, $\mathrm{R} 1$ [for 2405 reflections with $\left.\mathrm{I}>2 \sigma(\mathrm{I})\right]=$ $0.028, w \mathrm{R}^{2}$ (for all data) $=0.068, \mathrm{~S}=1.06$, largest diff. peak and hole $=0.266 \mathrm{eA}^{-3} /-0.245 \mathrm{eA}^{-3}$.

Crystallographic data (excluding structure factors) for structure reported in this work have been deposited with Cambridge Crystallographic Data center as supplementary publication no 1048447 (3a) Copies of the data can be obtained free of charge on application to the Director, CCDC, 12 Union Road, Cambridge CB2 1EZ, UK (fax: +44(1223) 336 033: e-mail: deposit@ccdc.cam.ac.uk.

\section{References}

1. Bharti, S. K.; Nath, G.; Tilak, R.; Singh, S. K. Eur. J. Med. Chem. 2010, 45, 651-660. https://doi.org/10.1016/j.ejmech.2009.11.008

2. Jung, Y.-K.; Kim, K.-S.; Gao, Z.-G.; Gross, A. S.; Melman, N.; Jacobson, K. A.; Kim, Y.-C. Bioorg. Med. Chem. 2004, 12, 613-623. https://doi.org/10.1016/j.bmc.2003.10.041

3. Welch, J. T. Tetrahedron 1987, 43, 3123-3197. https://doi.org/10.1016/S0040-4020(01)90286-8

4. Singh, G. S.; D’hooghe, M.; De Kimpe, N. Tetrahedron 2011, 67, 1989-2012. https://doi.org/10.1016/j.tet.2011.01.013

5. Moody, C. J.; Hughes, R. A.; Thompson, S. P.; Alcaraz, L. Chem. Commun. 2002, 17601761. https://doi.org/10.1039/B204868J

6. Crews, P.; Kakou, Y.; Quinoa, E. J. Am. Chem. Soc. 1988, 110, 4365-4368. https://doi.org/10.1039/B204868J

7. Shinagawa, H.; Yamaga, H.; Houchigai, H.; Sumita, Y.; Sunagawa, M. Bioorg. Med. Chem. 1997, 5, 601-621. https://doi.org/10.1016/S0968-0896(96)00273-8 
8. Shivarama Holla, B.; Malini, K. V.; Soorryanarayana Rao, B.; Sarojini, B. K.; Suchetha Kumari, N. Eur. J. Med. Chem. 2003, 38, 313-318. https://doi.org/10.1016/S0223-5234(02)01447-2

9. Gorczynski, M. J.; Leal, R. M.; Mooberry, S. L.; Bushweller, J. H.; Brown, M. L. Bioorg. Med. Chem. 2004, 12, 1029-1036.

https://doi.org/10.1016/j.bmc.2003.12.003

10. Helal, C. J.; Sanner, M. A.; Cooper, C. B.; Gant, T.; Adam, M.; Lucas, J. C.; Kang, Z.; Kupchinsky, S.; Ahlijanian, M. K.; Tate, B.; Menniti, F. S.; Kelly, K.; Peterson, M. Bioorg. Med. Chem. Lett. 2004, 14, 5521-5525.

https://doi.org/10.1016/j.bmcl.2004.09.006

11. Hang, P. C.; Honek, J. F. Bioorg. Med. Chem. Lett. 2005, 15, 1471-1474. https://doi.org/10.1016/j.bmcl.2004.12.076

12. Heal, B. C. W. In Comprehensive Heterocyclic Chemistry III; Joule, J. A., Ed.; Katritzky, A. R., Ramsden, C. A., Scriven, E. F. V., Taylor, R. J. K., Eds.; Elsevier: Oxford, U.K., 2008; Vol. 4, Chapter 4.06, pp 635-754.

13. Koutentis, P. A.; Ioannidou, H. A. In Science of Synthesis; Schaumann, E.; Ed.; Georg Thieme Verlag KG: Stuttgart, Germany, 2010; Vol. 11, Product Class 18, pp 267-391.

14. Thompson, M. J.; Heal, W.; Chen, B. Tetrahedron Lett. 2006, 47, 2361-2364. https://doi.org/10.1016/j.tetlet.2006.02.004

15. Shimada, Y. Japan Kokai Tokkyo Koho CODEN; JXXAF JP 02113070 A2 19900425; Japan; Chem. Abstr. 1990, 113, 99405x.

16. Ugi, I. Angew. Chem. Inter. Ed. Engl. 1962, 1, 8-21. https://doi.org/10.1002/anie.196200081

17. Dömling, A.; Ugi, I. Angew. Chem. Intern. Ed. 2000, 39, 3168-3210.

18. Marcaccini, S.; Torroba, T. Nat. Protoc. 2007, 2, 632-639. https://doi.org/10.1038/nprot.2007.71

19. Thompson, M. J.; Chen, B. Tetrahedron Lett. 2008, 49, 5324-5327. https://doi.org/10.1016/j.tetlet.2008.06.067

20. Gomaa, M. A.-M.; Hassan, A. A.; Shehatta, H. S. Heteroatom Chem. 2006, 17, 261-266. https://doi.org/10.1002/hc.20198

21. Hassan, A. A.; El-Shaieb, K. M. A.; Mohamed, N. K.; Tawfeek, H. N.; Bräse, S.; Nieger, M. Tetrahedron Lett. 2014, 55, 2385-2388.

https://doi.org/10.1016/j.tetlet.2014.02.107

22. Kalinowski, H. O.; Berger, S.; Brann, S. ${ }^{13}$ C NMR Spektroskopic; Georg Thieme Verlag; Stuttgart, 1984, p. 121.

23. Gewald, K.; Schindler, R. J. Prak. Chem.-Chemiker Zeitung 1990, 332, 223-228.

24. Hassan, A. A.; Mohamed, N. K.; Shawky, A. M.; Döpp, D. Arkivoc 2003, (i), 118-128.

25. $\quad$ Lee, B. W.; Lee, S. D. Tetrahedron Lett. 2000, 41, 3883-3886. https://doi.org/10.1016/S0040-4039(00)00493-7 
26. Zaharia, V.; Ignat, A.; Palibroda, N.; Ngameni, B.; Kuete, V.; Fokunang, C. N.;

Moungang, M. L.; Ngadjui, B. T. Eur. J. Med. Chem. 2010, 45, 5080-5085.

https://doi.org/10.1016/j.ejmech.2010.08.017

27. Pluijgers, C. W.; Sijpesteijna, A. K. Ann. Appl. Biol. 1966, 57, 465-473.

https://doi.org/10.1016/j.ejmech.2010.08.017

28. Forster, M. O.; Saville, W. B. J. Chem. Soc. Trans. 1920, 117, 753-761. https://doi.org/10.1039/CT9201700753

29. Parikh, P. M.; Deliwali, C. V. Indian J. Chem. 1965, 3, 45-46.

30. Vogel, A. I. A. Text Book of Practical Organic Chemistry $3^{\text {rd }}$ (ed.) Longman, London, 1957.

31. Organikum "Organisch Chemistry Grundpraktikum" VEB Deutscher Verlag der Wissenschaften, Berlin 1973.

32. Sheldrick, G. M. Acta Crystallogr., Sect. A 2008, A64, 112-122.

https://doi.org/10.1107/S0108767307043930

33. Sheldrick, G. M. Acta Crystallogr., Sect. C 2015, C71, 3-8.

https://doi.org/10.1107/S2053229614024218 